\title{
A Robust Real-Time Face Tracking using Head Pose Estimation for a Markerless AR System
}

\author{
Márcio C. F. Macedo*†, Antônio L. Apolinário Jr.*, Antonio C. S. Souza ${ }^{\dagger}$ \\ *Departamento de Ciência da Computação \\ Universidade Federal da Bahia (UFBA) \\ 40170-970 - Salvador - BA - Brazil \\ ${ }^{\dagger}$ Laboratório de Realidade Aumentada, Jogos Digitais e Dispositivos Móveis (LABRAGAMES) \\ Departamento de Tecnologia em Eletro-eletrônica \\ Instituto Federal de Educação, Ciência e Tecnologia da Bahia (IFBA) \\ 40301-015 - Salvador - BA - Brazil
}

\begin{abstract}
In this paper we present an extension to the KinectFusion algorithm that allows a robust real-time face tracking. This is achieved altering the original algorithm such that when the tracking algorithm fails, it uses a head pose estimation to give an initial guess to the Iterative Closest Point (ICP) algorithm. We show that this approach can handle more face pose changes and variations than the original KinectFusion's tracking.
\end{abstract}

Index Terms-Augmented Reality; Head Pose Estimation; Face Tracking.

\section{INTRODUCTION}

Augmented reality (AR) is a technology in which a user's view of a real scene is augmented with additional virtual information. Accurate tracking, or camera pose estimation, is required for the proper registration of virtual objects. However, tracking is one of the main technical challenges of AR.

In some AR systems, the user turns his head in front of a camera and the head is augmented with a virtual object. In this case, is desirable an algorithm able to track the person's head with enough accuracy and in real-time. One way to achieve this goal is building a reference 3D model of the user's head and aligning it to the current head captured by the sensor.

We present an approach for robust real-time face tracking based on head pose estimation for a markerless AR system. First, a reference 3D model is built with a $3 \mathrm{D}$ reconstruction system. Afterward, the Kinect raw data is aligned to the reference 3D model, predicting the current camera pose. Finally, to improve the robustness of the system, is used a head pose estimator to give an initial guess to the tracking algorithm when it fails. An overview of this method can be seen in Figure 1 .

The method is inspired by two recent works: An algorithm that allows the dense mapping of extended scale environments in real-time using only Kinect raw data called KinectFusion [1], and an algorithm for estimating the location and orientation of a person's head from low quality depth data [2]. Our approach adapts the KinectFusion to reconstruct heads and extends its tracking using the head pose estimation. We show that this approach can handle more face pose changes and variations than the original KinectFusion's tracking.
The rest of the paper is arranged as follows. Section 2 provides a review on the related work of surface reconstruction, markerless AR and real-time head pose estimation. Section 3 presents the proposed algorithm. Section 4 discusses the experimental results. The paper concludes in Section 5, with a summary and discussion of future work.

\section{RELATED WORK}

Surface reconstruction, markerless AR and head pose estimation have been driven by different approaches, as we can see in the next subsections.

Surface reconstruction: In 1996, Curless and Levoy [3] described a method for volumetric integration of complex models from range images (VRIP). The volumetric integration basically consists of a cumulative weighted signed distance function (SDF). This method is able to integrate high-detail models, in the order of a million triangles. However, the execution time can be in the order of hours and it is not suitable for AR applications. The range images used in this work were captured by laser scanners. Laser scanners provide range images with high accuracy, but the drawback of them is the high cost of the hardware.

In 2002, Rusinkiewicz et al. [6] described a method for real-time 3D model acquisition. Using a real-time low-quality structured-light 3D scanner, they aligned the range images from different viewpoints to produce complete 3D rigid objects. Different from the method proposed by Curless and Levoy, it operated at $\approx 10 \mathrm{~Hz}$ with lower cost hardware but did not reconstruct high-quality models. It was the first system to reconstruct and display the 3D models in real-time and it increased the possibility to do markerless AR with surface reconstruction.

In 2010, Cui et al. [7] described a method for 3D object scanning using a time-of-flight (ToF) camera. In this work, Cui et al. showed a superresolution method that improves significantly the quality of the depth maps acquired from a ToF camera. One drawback of this method is that it does not run in real-time. Compared to the other scanners presented, time-of-flight cameras have the lowest cost and provide range images with the lowest accuracy. 


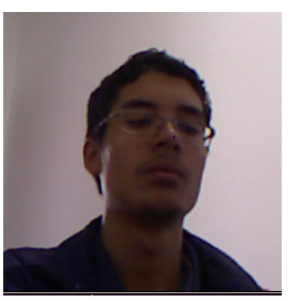

Live Stream

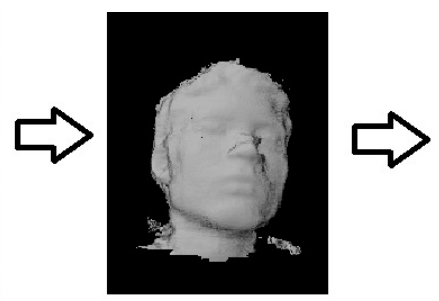

Reference 3D Model

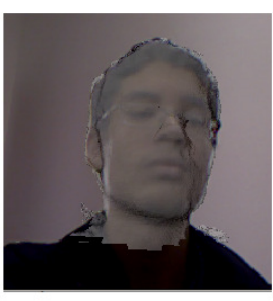

AR with the user's head

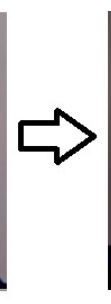

Fast Motion and ICP Failure

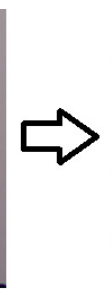

ICP Recovery with Head Pose Estimation

Fig. 1. Overview of the online processing pipeline. A) RGB-D live stream. B) Reference 3D model is reconstructed with KinectFusion. C) The user's head is augmented with a virtual object and the current camera pose is predicted by the alignment between the reference 3D model and the Kinect raw data. D) The user rotated his face fast and the ICP failed. E) The head pose estimation is used to give an initial guess to the ICP and the fast motion is compensated.

Markerless AR: In 1999, in the field of AR, Kato and Billinghurst [4] presented a video-based AR system with marker tracking which mixed virtual images on the real world. They used fast and accurate computer vision techniques to track the fiducial markers through the video. The system presented is also called ARToolKit and it is one of the most used systems in this field.

In 2000, Simon et al [5] described one of the first methods using markerless tracking for an AR system: a tracker of planar structures. Despite being a special case of tracking (i.e. when there is a planar structure visible in the scene), the method does not need fiducial markers and robustly track the planar structures through the video.

Surface Reconstruction + Markerless AR: In 2011, Izadi et al. [1] described a system that enables real-time detailed 3D reconstruction of a scene using the depth stream from a Kinect. The system was called KinectFusion. Using a GPU, it was the first system to reconstruct high-detail models at $\approx 30 \mathrm{~Hz}$. Izadi et al. [1] also presented some markerless AR applications, showing the level of the user interaction in their system. As mentioned before, our approach uses the KinectFusion to reconstruct heads and extends its tracking algorithm taking advantage of the model that we are reconstructing.

In the same year, Weise et al. [8] presented a system that enables active control of facial expressions of a digital avatar in real-time. The system is called FaceShift [9]. It was the first system to enable high-quality reconstruction and control of facial expressions using blendshape representation in realtime. FaceShift represents a great advance in the field of markerless AR and non-rigid surface reconstruction.

Head Pose Estimation: Recently, automatic real-time 3D head pose estimation have become popular due to the increasing availability of the 3D scanners.

Breitenstein et al. [10] developed a real-time algorithm to estimate 3D head pose using GPUs. Using high-quality depth data, the algorithm computes a set of candidate nose positions and compares the input depth data to precomputed pose images of an average face model.

Fanelli et al. [2] developed a real-time algorithm to estimate 3D head pose using only the CPU. Using low-quality depth data (e.g. captured from a Kinect sensor), the algorithm trains random forests to estimate head pose.

We choose this last algorithm for head pose estimation because it operates directly on low-quality depth data.

\section{Robust ReAl-Time FACE Tracking using HeAD POSE ESTIMATION}

In this section we describe the proposed improvements we made to the KinectFusion's tracking algorithm to track and reconstruct faces. Before, we describe the original KinectFusion and the head pose estimation used.

\section{A. Reconstructing 3D Models with KinectFusion}

KinectFusion [1] is a system that integrates raw depth data from a Kinect camera into a voxel grid to produce a highquality $3 \mathrm{D}$ reconstruction of a scene. The system first applies a bilateral filter [11] to the depth map to reduce the noise preserving discontinuities of the raw data. The filtered depth map is then converted into a vertex map and a normal map. To compute the transformation that defines the camera pose is used a real-time point-plane variant of the well known ICP (Iterative Closest Point) algorithm [12]. The ICP estimates the transformation that aligns the current depth frame with the accumulated model. Once with the current transformation, the raw depth data can be integrated into the voxel grid. The grid stores at each voxel the distance to the closest surface and a weight that indicates uncertainty of the surface measurement. This distance is a truncated signed distance function (TSDF). Surface extraction is achieved by detecting zero-crossings through a raycaster. All these operations are made using the GPU. An overview of this method can be seen in Figure 2 .

\section{B. Real-Time Head Pose Estimation from Consumer Depth Cameras using Random Regression Forests}

Random Regression Forests are trees trained randomly that generalize a problem better than decision trees taken separately [13]. Fanelli et al. [2] trained random forests to estimate head pose from low-quality depth images. To train the trees, each depth map was annotated with labels indicating head center and Euler rotation angles. These labels were estimated automatically using ICP after a 3D facial reconstruction. After the labeling and training, the head pose can be estimated letting every image region to vote it. The vote consists of a 


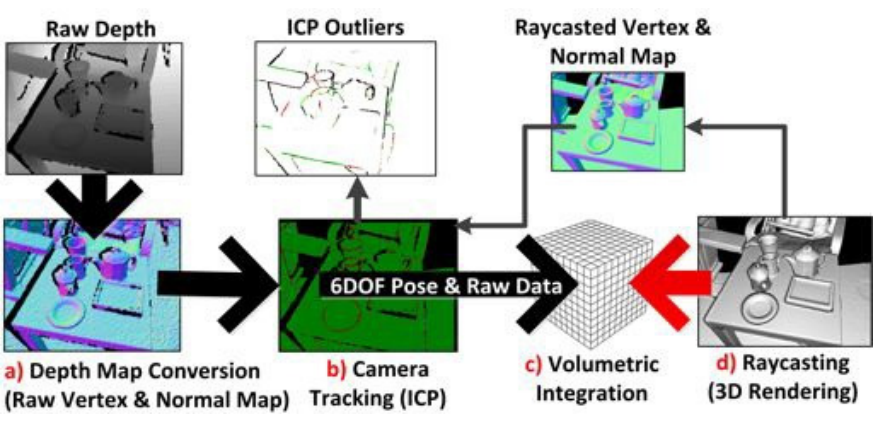

Fig. 2. Overview of KinectFusion's pipeline [1].

classification whether the image region contains a head and a retrieval of a Gaussian distribution computed during the training and stored at the leaf. This probabilistic approach achieves high accuracy and runs in real-time using only CPU.

\section{Our Approach}

The system we build consists of two main stages: head reconstruction and markerless AR face tracking. The first stage consists in the application of KinectFusion to reconstruct the user's head (Figure 3) and the second stage consists in tracking of the user's face augmented with a virtual object. We use the tracking algorithm in these two stages.

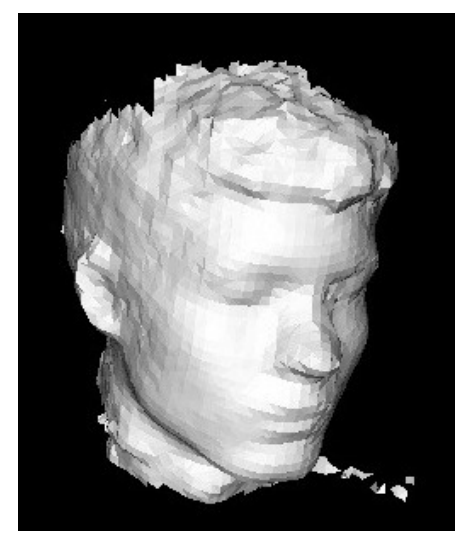

Fig. 3. An example of user's head reconstructed with the KinectFusion.

For each new depth frame $D$, we segment the region of interest (i.e. user's head) by applying a Z-axis threshold of $1.3 \mathrm{~m}$. It is the maximum acceptable distance from the user's head to the camera center in the original Fanelli's head pose estimation [2]. After that, we apply the ICP algorithm to compute the current camera pose (i.e. transformation matrix $T)$. The ICP uses the projective data association [14] to find correspondences between the current depth frame and the accumulated model. In this association, each point is transformed into camera coordinate space and perspective projected into image coordinates. The corresponding points are that on the same image coordinates. The ICP fails (i.e. does not converge to a correct alignment) when there is not a small pose variation between sequential frames. In this case, we use the head pose estimation to give a new initial guess to the ICP compute correctly the current transformation.

The use of the head pose estimation is shown in Algorithm 1. Given the previous depth frame $D_{\text {prev }}$ and the current depth frame $D_{\text {curr }}$, the head pose estimation is used to set the head orientation ( $R_{\text {prev }}$ and $\left.R_{\text {curr }}\right)$ and the head center $\left(H c_{\text {prev }}\right.$ and $H c_{c u r r}$ ) of them. The head centers are converted from camera to global coordinates. The incremental rotation matrix $R_{\text {inc }}$ and the translation $\Delta t$ between the previous and the current head center are computed (lines 7 and 8). The translation $\Delta t$ is added to the current global translation $t$ (line 9). The implicit surface is then raycasted to generate a new view (i.e. new previous depth frame) (line 10). The raycasted view is rotated around $H c_{c u r r}$ with $R_{i n c}$ (line 11). Finally, we reuse the ICP to estimate the current $T$.

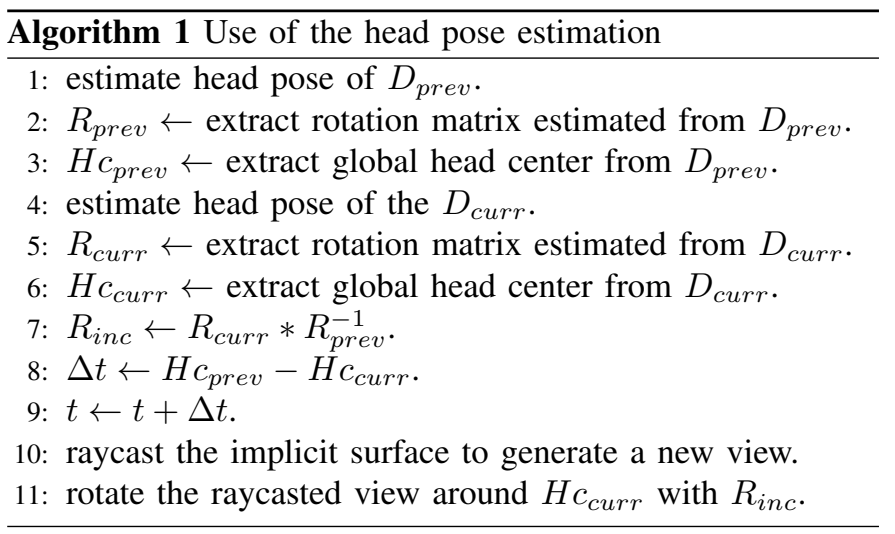

\section{RESULTS AND Discussion}

In this section we analyze the system's performance and describe the experimental setups we used.

We based our system on the open source $\mathrm{C}++$ implementation of the KinectFusion [15] released by the PCL project [16] and on the open source $\mathrm{C}++$ implementation of the head pose estimation released by Fanelli [17]. For all tests we ran our system on an Intel(R) Core(TM) i7-3770K CPU @ $3.50 \mathrm{GHz}$ 8GB RAM in real-time. When the head pose estimation was used, the main pipeline of our system needed only $80 \mathrm{~ms}$ to process a frame.

We tested our algorithm with real data captured with a Kinect sensor using a grid with volume size of $5 \mathrm{~cm} \times 5 \mathrm{~cm} \times 13 \mathrm{~cm}$ that could reconstruct high-quality heads. We can analyze the qualitative performance for two cases: fast translation and rotation of the user's face.

When the user translated his face in front of the camera and the ICP failed, the algorithm could give a correct initial guess to the ICP. If the user translates his face fast, there will not be sufficient points at the same image coordinates and the ICP will fail. By applying our approach we can solve this problem. This situation can be seen in Figure 4

The algorithm slightly improved the tracking performance when the user rotated his face and the ICP failed. The reason is that the larger the pose variation, the larger the nonoverlapping region, and there are cases that the ICP is not 

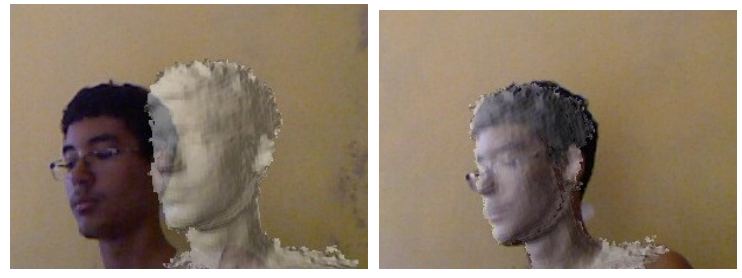

Fig. 4. A) The user translated his face fast. A small number of points were at the same image coordinates and the ICP failed. B) By applying our approach we solved this problem.

appropriate in the presence of non-overlapping regions (Figure 1. $\mathrm{D}$ and $\mathrm{E}$ ) even if the head pose estimation provides the initial guess. In this case (Figure 5), the user needs to reposition his face to the tracking algorithm align correctly the raw depth data.

The accuracy of the head pose estimation is the same as the Fanelli's approach (angle error: about $8^{\circ}$ in each axis; head center error: $10 \mathrm{~mm}$ ). However, as mentioned before, in the case of large pose variations, its initial guess is not sufficient for the ICP algorithm.

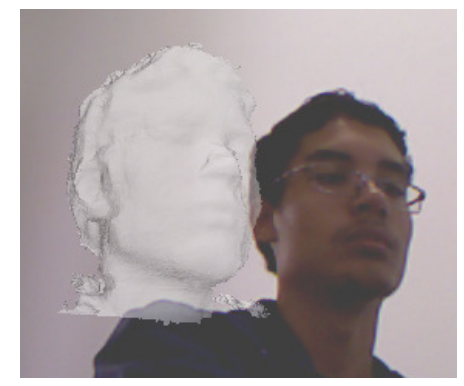

Fig. 5. An example of tracking failure. The user needs to reposition his face to the tracking algorithm align correctly the raw depth data to the reference 3D model.

\section{CONCLUSIONS AND Future Work}

We presented an approach for robust real-time face tracking using head pose estimation for a markerless AR system. We used the KinectFusion to reconstruct the user's head and we extended its tracking algorithm using the head pose estimation to give the initial guess to the ICP algorithm when it failed. We showed that this approach can handle more face pose changes than the original KinectFusion's tracking and the use of the head pose estimation is suitable for AR applications, as it runs in real-time.

Encouraged by the work of Meister et al. [18], for future work we plan to analyse the accuracy of the system to check if this method can be used for medical applications. Further improvements can be achieved by implementing a deformable registration to track the face, as it is a non-rigid object, or extending this to other objects by the use of other pose estimators.

\section{ACKNOWLEDGMENTS}

We are grateful to Gabriele Fanelli and the PCL project for providing the open-source implementations. This research is financially supported by FAPESB and CAPES.

\section{REFERENCES}

[1] S. Izadi, D. Kim, O. Hilliges, D. Molyneaux, R. Newcombe, P. Kohli, J. Shotton, S. Hodges, D. Freeman, A. Davison, and A. Fitzgibbon, "Kinectfusion: real-time $3 \mathrm{~d}$ reconstruction and interaction using a moving depth camera," in Proceedings of the 24th annual ACM symposium on User interface software and technology, ser. UIST '11, New York, NY, USA, 2011, pp. 559-568.

[2] G. Fanelli, T. Weise, J. Gall, and L. V. Gool, "Real time head pose estimation from consumer depth cameras," in 33rd Annual Symposium of the German Association for Pattern Recognition (DAGM'11), September 2011.

[3] B. Curless and M. Levoy, "A volumetric method for building complex models from range images," in Proceedings of the 23rd annual conference on Computer graphics and interactive techniques, ser. SIGGRAPH '96. New York, NY, USA: ACM, 1996, pp. 303-312.

[4] H. Kato and M. Billinghurst, "Marker tracking and hmd calibration for a video-based augmented reality conferencing system," in Augmented Reality, 1999. (IWAR '99) Proceedings. 2nd IEEE and ACM International Workshop on, 1999, pp. $85-94$.

[5] G. Simon, A. Fitzgibbon, and A. Zisserman, "Markerless tracking using planar structures in the scene," in Augmented Reality, 2000. (ISAR 2000). Proceedings. IEEE and ACM International Symposium on, 2000, pp. 120 -128 .

[6] S. Rusinkiewicz, O. Hall-Holt, and M. Levoy, "Real-time 3d model acquisition," in Proceedings of the 29th annual conference on Computer graphics and interactive techniques, ser. SIGGRAPH '02. New York, NY, USA: ACM, 2002, pp. 438-446.

[7] Y. Cui, S. Schuon, D. Chan, S. Thrun, and C. Theobalt, "3d shape scanning with a time-of-flight camera," in Computer Vision and Pattern Recognition (CVPR), 2010 IEEE Conference on, june 2010, pp. 1173 -1180 .

[8] T. Weise, S. Bouaziz, H. Li, and M. Pauly, "Realtime performance-based facial animation," in ACM SIGGRAPH 2011 papers, ser. SIGGRAPH '11. New York, NY, USA: ACM, 2011, pp. 77:1-77:10.

[9] (2013, Jan.) Faceshift. [Online]. Available: http://www.faceshift.com/

[10] M. Breitenstein, D. Kuettel, T. Weise, L. Van Gool, and H. Pfister, "Realtime face pose estimation from single range images," in Computer Vision and Pattern Recognition, 2008. CVPR 2008. IEEE Conference on, june 2008, pp. $1-8$.

[11] C. Tomasi and R. Manduchi, "Bilateral filtering for gray and color images," in Computer Vision, 1998. Sixth International Conference on, jan 1998, pp. $839-846$.

[12] P. Besl and H. McKay, "A method for registration of 3-d shapes," Pattern Analysis and Machine Intelligence, IEEE Transactions on, vol. 14, no. 2, pp. $239-256$, feb 1992.

[13] L. Breiman, "Random forests," in Machine Learning, 2001, pp. 5-32.

[14] S. Rusinkiewicz and M. Levoy, "Efficient variants of the icp algorithm," in 3-D Digital Imaging and Modeling, 2001. Proceedings. Third International Conference on, 2001, pp. 145 -152.

[15] (2013, Mar.) Kinfu. [Online]. Available: http://svn.pointclouds.org/pcl/ trunk/gpu/kinfu/

[16] R. Rusu and S. Cousins, " $3 \mathrm{~d}$ is here: Point cloud library (pcl)," in Robotics and Automation (ICRA), 2011 IEEE International Conference on, may 2011, pp. $1-4$.

[17] (2013, Mar.) Fanelli's home page. [Online]. Available: http://www. vision.ee.ethz.ch/ $\sim$ gfanelli/

[18] S. Meister, S. Izadi, P. Kohli, M. Hämmerle, C. Rother, and D. Kondermann, "When can we use kinectfusion for ground truth acquisition?" in Workshop on color-depth fusion in robotics, IEEE/RSJ International Conference on Intelligent Robots and Systems, 2012. 